\title{
Periodically tailored titania nanotubes for enhanced drug loading and releasing performances
}

\author{
Karan Gulati', Krishna Kant ${ }^{\mathrm{a},}$, David Findlay ${ }^{\mathrm{b}}$, Dusan Losic ${ }^{\mathrm{a} *}$ \\ ${ }_{5}$ Received (in XXX, XXX) Xth XXXXXXXXX 20XX, Accepted Xth XXXXXXXXX 20XX \\ DOI: $10.1039 / b 000000 x$
}

The structural engineering of titania nanotubes (TNTs) using electrochemical anodization was performed to generate periodically modulated (p-TNTs) internal structures by applying an oscillatory voltage during anodization process to demonstrate their improved drug-loading and drug releasing properties. Drug

10 loading and in-vitro drug release studies compared with conventional TNTs with flat structures suggested considerable improvement with increased drug loading, reduced burst release and extended drug release for over 2 weeks. Furthermore, layer of p-TNTs arrays were fractured by ultrasonication into liberated TNTs capsules and their potential applications as drug micro/nano-carriers for targeted and localized drug delivery is proposed. The presented electrochemical approach for structural engineering of TNTs provides

15 new prospects in designing TNTs drug releasing implants with advanced drug-loading/release characteristics for localized drug delivery. Such implant modifications can further be tailored to cater various implant challenges and bone therapies like: inflammation, infection and poor implant integration.

\section{Introduction}

Each year several million cases of bone complications (severe 20 fractures, osteoporosis, bone infection etc.) are reported worldwide that demand extensive therapeutic action and surgery [1]. These conditions are generally treated via prolonged systemic administration of drugs/hormones, bone cement placement and in severe situations: grafting, implantation and amputation. Bone 25 implants made of $\mathrm{Ti}$ and its alloys are used to fix severely fractured bone fragments via insertion of Ti plates and screws. These implants often fail due to extreme inflammatory response, high incidence of bacterial infection and poor bone-implant bonding (osseointegration) [2-3]. Therapeutics delivered via 30 systemic route are unable to reach the target site in effective concentration. Hence, local therapeutic elution directly from the implant surface is regarded as an emerging solution to address these issues.

Research aiming at improving current bone implant 35 technology mostly investigates titanium surfaces as it is the most successful biomaterial for bone applications, due to its corrosion resistance, biocompatibility and appropriate mechanical strength [4]. These studies are primarily focused to improve properties of titanium bone implants by micro/nanoengineering of their surface 40 to enhance roughness or to coat active molecules or drugs to promote osseointegration and prevent bacterial invasion [5-6].

${ }^{a}$ School of Chemical Engineering, The University of Adelaide, Adelaide, SA 5005, Australia.E-mail: dusan.losic@adelaide.edu.au

$45^{\mathrm{b}}$ Discipline of Orthopaedics \& Trauma, University of Adelaide, Australia
Although surface roughening has been reported to enhance bone cell activity; they are unable to modulate immune responses and promote appropriate bone functions that will ensure implant integration without extensive inflammation [7-8]. Furthermore,

50 coating of drugs/proteins on titanium has poor release kinetics; and with the aid of non-biodegradable there exists risk of toxic degradation products [9]. These early attempts on titanium modification did not effectively cater towards most common implant needs: modulating immune reactions, preventing

55 bacterial invasion and promoting osseointegration. Therefore, an advanced surface engineering of titanium implant's surface with generated nanostructures, able to provide drug loading/release capability is recognized as a desirable approach to advance performance of bone implants with minimized rejection and 60 improved bone healing.

Nanotubular titania or titania nanotube [TNTs] arrays generated on titanium surface using simple electrochemical anodization process, has recently emerged as a promising solution to address these limitations and aim towards improving 65 existing medical implants [10]. The generated TNT layer is composed of an array of hollow cylindrical nanotubes vertically aligned on the titanium surface through unique self-ordered electrochemical process. In comparison with micro or nano-rough titanium, the TNTs offer increased surface area and 70 biocompatibility, tailorable dimensions, surface chemistry and ability to load and release therapeutics in controllable fashion, which makes them ideal for development of drug-releasing implants [9]. Therefore, TNTs on titanium surfaces are the most 
suitable nano-engineered modification to enable localized drug delivery (LDD); to address some problems of systemic drug delivery such as low efficacy, poor bio-distribution, lack of selectivity, drug overdose and toxicity in non-target tissues [95 10]. To date TNTs have been successfully explored for loading and hence releasing many drugs including: antibiotics, antiinflammatory, anticancer drugs and osseointegrating and bone forming proteins [10]. Osseointegrating abilities of the TNTs have also been confirmed by various in-vitro and in-vivo 10 investigations [8,12]. It has also been proven that TNTs contribute towards bone cell responses in two ways: nanotube dimensions improve bone cell adhesion and the spacing between nanotubes can provide a pathway for nutrients to reach cells once implant has integrated with the bone [13]. All these studies 15 suggest excellent potential of TNTs for localized drug delivery application and bone therapy.

When TNTs come into contact with the physiological solution inside the host body, the drug release from nanotubes is controlled by a diffusion process governed by a number of factors 20 such as the molecular size and charge of drugs, the dimensions of nanotubes (diameter and length), their charge and surface chemistry, interaction between drug molecules and nanotube surface, the dissolution rate of drugs, diffusion coefficient, $\mathrm{pH}$ and so on. In order to control the release of drugs from TNTs 25 various approaches have been explored including: structural modifications of TNTs dimensions (diameter and length), surface chemistry, and reduction of the pore openings by polymer deposition [9-11]. Controlling TNTs dimensions such as nanotube diameters and length is reported as the simplest 30 approach to control the drug loading amount and the release kinetics, but with considerable limitations [10,14,15]. These studies have demonstrated that the release of several model drugs (of varied chemistries and catering different conditions) from TNTs can be extended by reducing the diameters and increasing 35 the lengths. This result is expected because drug molecules are trapped deeper inside the nanotubes and a longer time is required to diffuse out of the TNTs. However, the drug loading is also influenced by the TNTs dimensions (i.e. less drug is loaded as the nanotube diameter is reduced), which indicates that this strategy 40 is limited to provide extended release of therapeutics over a short period of time.

To address these limitations; alternative approaches have been reported by our group and others such as: thin bio-polymer coating on drug loaded TNTs, micelle encapsulated drugs inside ${ }_{45}$ TNTs, and covalently tethered antibiotics [15-20]. Significant improvements with sustained drug release of 4-8 weeks with zero order kinetics were achieved which are desirable performances in medical therapy. However, these methods compared with simplicity of structural modification approach showed some

${ }_{50}$ disadvantages including: reproducibility and optimisation of polymeric degradation, the complexity of polymer micelle synthesis and covalent attachment procedures which are difficult to be applied in clinical environment. Therefore, the structural modification approach of TNTs is still considered as a valuable 55 approach, which requires further improvement and development.

In this work we present a new concept for improving drug loading and release characteristics of TNTs by structural engineering of their nanotube structures. This is achieved by generation of periodically shaped morphology of nanotubes using 60 an altered periodic voltage during the anodization process. The aims are: (1) to demonstrate the synthesis of new type of TNTs with periodically modulated internal structures using cyclic anodization, (2) to prove their advanced drug-loading and drug releasing properties relevant for their applications as drug${ }_{65}$ releasing implants and, (3) to introduce the preparation of titania nanotubes capsules from these structures as potential nano- and micro-carriers for drug delivery applications. We propose that TNTs with periodic modulated structures due their characteristic geometry will alter diffusion process of loaded drugs inside 70 nanotubes and slow their release kinetics. The method is expected to provide significant advances in designing TNTs drug releasing implants compared with previous reports where structural modifications were performed on TNTs with flat structures by altering their diameters and length $[14,15]$. The concept of the 75 fabrication of TNTs with periodically shaped structures using cyclic anodization voltage is presented in Scheme 1. This anodization approach is extended from our previous work on tailoring pore structures of nanoporous alumina (NPA) [21]. The idea is based on periodic changes of anodization voltage from ${ }_{80}$ higher voltage $(100 \mathrm{~V})$ which provide the large pore diameters to lower voltage $(60 \mathrm{~V})$ where the pore diameters are reduced [22]. These changes of anodization conditions are proposed to make periodic modulations of internal diameters of TNTs and advance their drug releasing performances compared with flat nanotube 85 structures. To prove the hypothesis, TNTs both with conventional TNT and periodically shaped (p-TNT) structures were loaded with a model anti-inflammatory drug and evaluated for drug loading and in-vitro release characteristics. Furthermore, this strategy is applied to obtain liberated TNTs capsules from p-TNT 90 arrays towards the preparation of new nano and micro-carriers for drug delivery applications.

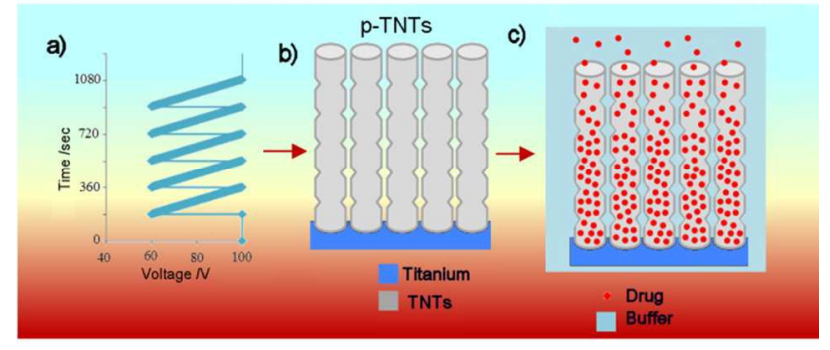

Scheme 1 Scheme of electrochemically engineered titania nanotubes on Ti substrate with periodically shaped titania nanotubes (p-TNTs) as drug95 releasing implants. (a-b) Voltage oscillatory signal $(60-100 \mathrm{~V})$ used for electrochemical anodization and fabrication of p-TNTs layer, (c) scheme of in-vitro drug release from nanotube structures.

\section{Experimental part}

\section{Materials and chemicals}

100 Titanium foil ( $99.6 \%$ purity, thickness $0.25 \mathrm{~mm}$ ) supplied by Sigma-Aldrich (Sydney, Australia) was used as substrate for preparation of TNTs. Ammonium fluoride, ethylene glycol and indomethacin were also obtained from Sigma-Aldrich (Sydney, Australia) and used as received. High purity Milli-Q water was 105 used for all experiments, which was ultrapure grade (18.2 M $\Omega$ ) 
sieved through a $0.22 \mu \mathrm{m}$ filter.

\section{Fabrication of titania nanotube (TNTs) arrays}

Titanium foil (cut in squares $1.2 \mathrm{~cm}$ ) was cleaned and polished mechanically using fine sandpaper and porous alumina powder in 5 order to reduce surface unevenness. The cleaned substrates were sonicated in acetone and water to further remove particle debris from the surface. The layer of TNTs was fabricated on the Ti foil using a two-step anodization procedure, using a specially designed electrochemical cell and a computer-controlled power 10 supply (Agilent), as described elsewhere [16]. The specially designed electrode holder allowed only a circular area of $\mathrm{Ti}$ substrate (diameter $1 \mathrm{~cm}$ ) to be exposed to electrolyte $(0.3 \%$ $\mathrm{NH}_{4} \mathrm{~F}$ and $3 \%$ water in ethylene glycol). Two steps anodization was employed for preparation of both TNTs with flat and 15 periodically modulated nanotube structures (p-TNTs). The first step was the same for both samples based on anodization at constant voltage of $100 \mathrm{~V}$ for $2 \mathrm{~h}$ at $20^{\circ} \mathrm{C}$. The formed TNTs layer was removed by sonication in methanol allowing the underlying Ti substrate to be templated in order to achieve better 20 ordering of nanotubes in the following step. To obtain TNTs with periodically modulated morphology the second anodization was performed by specifically designed saw-like cyclic voltage signal which includes two steps: increasing ramp (60-100 V) and fast drop $(100$ to $60 \mathrm{~V})$. These two voltages are selected based on our ${ }_{25}$ previous work and literature data showing the voltage dependence of pore diameters [21]. For the preparation of pTNTs $2^{\text {nd }}$ anodization was split into three steps: (1) anodization at constant voltage $100 \mathrm{~V}$ for $10 \mathrm{~min}$, (2) periodic cyclic anodization involving voltage drop ( 100 to $60 \mathrm{~V}$ ) and ramp (60 to $30100 \mathrm{~V}$ ) in $3 \mathrm{~min}$ cycles (repeated 15 times), and (3) last anodization step with constant $100 \mathrm{~V}$ for $10 \mathrm{~min}$. The applied voltage graph during whole process is shown in Fig. 2. This unique combination of constant voltage, and cyclic anodization was selected to create initial part of TNT identical to 35 conventional TNTs prepared constant voltage used as control. The second anodization step for the preparation of TNTs with flat nanotubes was performed using only $100 \mathrm{~V}$ and anodization time of $1 \mathrm{~h}$ which we found is required to have the same thickness of TNTs layer compared with p-TNTs. The voltage and current 40 signals with time were continuously monitored and recorded during anodization process using Labview software (National Instruments, USA)

\section{Preparation of liberated TNTs nanocapsules}

To liberate nanotube capsules from prepared p-TNTs we initially 45 explored several anodization procedures using different cyclic voltages (from $100 \mathrm{~V}$ to $20 \mathrm{~V}$ ) and signal patterns (saw, triangular, and rectangular) in order to obtain 'breaking points' or 'weak spots' between nanotube segments for their easy liberation. The rectangular voltage signal without any ramp, composed of $100 \mathrm{~V}$ 50 with time of $5 \mathrm{~min}$ followed by $20 \mathrm{~V}$ with time of $1 \mathrm{~min}$ was found as the most efficient method. The use of lower anodization voltage $(20 \mathrm{~V})$ is proposed to make barrier layer between $100 \mathrm{~V}$ anodized segments thinner and easily detachable from structure. The fabricated p-TNTs under these conditions were sonicated in 55 methanol to achieve their delamination from the underlying $\mathrm{Ti}$ substrate and detachment between individual nanotubes. This facilitated breakage of nanotubes into smaller fragments.
Liberated nanotube capsules and their bundles dispersed in methanol were purified using centrifugation and later 60 decantation/drying was performed to obtain nanotube capsules in a powder form.

\section{Drug loading in TNTs}

Hydrophobic anti-inflammatory drug indomethacin was selected as the model drug and dissolved in ethanol $(1 \% \mathrm{w} / \mathrm{v})$ prior to ${ }_{65}$ loading. Both nanotube surfaces (TNTs and p-TNTs) were cleaned using deionised water and dried in nitrogen. For drug loading into the nanotubes the active area of nanotubes, concentration/volume of the drug solution, time of loading/drying and temperature were maintained constant for both the samples. $7020 \mu \mathrm{l}$ of the drug solution was pipetted onto the nanotube surface and upon drying, the surfaces were gently wiped using a soft tissue in order to remove excess drug accumulated on the surface. Loading, drying and wiping steps were repeated several times in order to load substantial amount of drug into the TNTs. Post75 loading the surfaces were flushed with equal volumes of phosphate buffer solution (PBS) and dried in air. This step washed any excess drug that was physisorbed on the upper surface of the nanotubes but not loaded inside them. Afterwards the dried drug loaded samples were characterised for the amount 80 of drug present in them.

\section{Characterisations}

\section{Quantitative analysis of drug loading}

To determine the amount of drug loaded in the nanotubes, thermo-gravimetric analysis (TGA) was performed. In order to 85 find the correct range of the drug decomposition, a known amount of pure drug was loaded into the platinum pan in TGA and heated in the furnace from $20{ }^{\circ} \mathrm{C}$ to $800{ }^{\circ} \mathrm{C}$, and its characteristic peak was obtained. Later the drug loaded TNT samples were characterised and the peak of the drug was 90 identified in order to calculate the correct amount of drug present. The drug loading experiments were repeated several times and the obtained values were averaged.

\section{In-vitro drug release characterisation}

95 The drug release characteristics of indomethacin loaded TNTs were obtained in in-vitro conditions in phosphate buffer solution (PBS) at $\mathrm{pH} 7.4$; by measuring the absorbance of released drug at 320nm using UV-Vis spectrophotometer (Cary 60, Agilent Technologies). Drug loaded flat TNTs and p-TNTs in separate 100 experimental setups were immersed in $5 \mathrm{ml}$ of $\mathrm{PBS}$, and at predetermined time intervals $3 \mathrm{ml}$ aliquot was drawn and transferred to a $3 \mathrm{ml}$ quartz cuvette (10 mm path length, Sterna Scientific, Australia) for absorbance analysis. At the same time fresh $3 \mathrm{ml}$ PBS was replaced back into the vials containing the 105 drug loaded TNTs. Absorbance measurements were performed every $10 \mathrm{~min}$ during $1^{\text {st }} 6 \mathrm{hrs}$, followed by measurement every 24 hrs until the entire drug amount was released into the PBS. The drug concentration for the corresponding absorbance values was calculated based on calibration curve for indomethacin in PBS. 110 Ultimately the concentration was expressed as weight \% (with $100 \%$ representing entire drug amount released in the PBS), and release profile for burst $\left(1^{\text {st }} 6 \mathrm{~h}\right)$ and delayed release was plotted with $\mathrm{Wt} \%$ of drug released Vs time. 

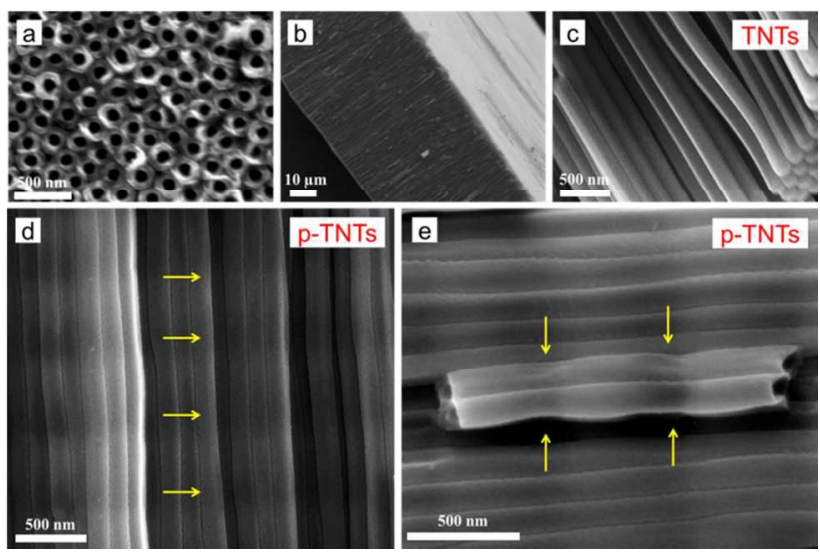

Fig. 1 Comparative SEM images of prepared TNTs with flat and periodically modulated structures (p-TNTs). Typical structure of (A) the top surface of nanotubes showing their open pores, (B) the cross-section of TNT layer showing the approximate length of the nanotubes, same for both flat TNTs and p-TNTs $(\sim 50 \mu \mathrm{m}),(\mathrm{C})$ cross-sectional image of flat TNTs showing typical structure of TNTs with well aligned and straight nanotubes, (D) cross-sectional SEM image of periodically modulated p-

10 TNTs depicting the alterations through its length which corresponds to periodic change of voltage during anodization, and (E) high resolution image of shaped p-TNTs showing periodic segments and indicates reduction of internal pore dimensions of the nanotubes.

\section{Results and discussion}

\section{${ }_{15}$ Structural characterisation of TNTs with periodically shaped structures}

Typical morphology of prepared TNTs with straight and periodically shaped nanotube structures as characterised by SEM is summarised in Fig. 1 showing their top and bottom surface, 20 and cross-sectional structure. The SEM image from the top surface (Fig. 1a) reveals nicely ordered and tightly packed nanotube structure with average nanotube diameter of $140 \pm 10$ $\mathrm{nm}$ which is the same for both TNTs and p-TNT substrates. Fig. 1b presents the cross-section of the nanotube film obtained after ${ }_{25}$ fracturing the TNT which was removed from the underlying Ti surface (only for imaging purpose). . The length for both nanotube structures was confirmed to be around $50 \mu \mathrm{m}$. The shapes of nanotubes for both TNTs and p-TNTs, determined from their fractured structures by cross-sectional imaging are presented 30 in Fig. 1c-e. The nanotubes on flat TNTs showed no differences across whole length of nanotubes structures; however nanotubes prepared by periodic altering of anodization voltage showed periodic changes of nanotubes structures at every $500-600 \mathrm{~nm}$ (Fig. 1d-e). This characteristic pattern relates to the increasing 35 and decreasing nanotube dimensions as result of periodic changing of voltage from $100 \mathrm{~V}$ to $60 \mathrm{~V}$, and is confirmed by close-up of a broken fragment of p-TNT in Fig. 1e.

These images represent outer nanotube surface morphology as the determination of diameters inside the nanotubes cannot be 40 achieved due to measurement limitations. However the outer and inner diameters correlate and hence it indicates that $\mathrm{p}$-TNTs offer varied diameters (outer and inner) along its length, which directly corresponds to periodic voltage oscillation. A similar observation was reported for nanoporous alumina fabricated under periodic 45 conditions $[21,23]$. Moreover the length of such periods on the $p-$ TNTs can be controlled by varying the time of the voltage ramp (data not shown). Furthermore, periodic TNT structures are reproducible and can be applied for modification of orthopaedic implants (plates, screws etc.) composed of Ti and its alloys. 50
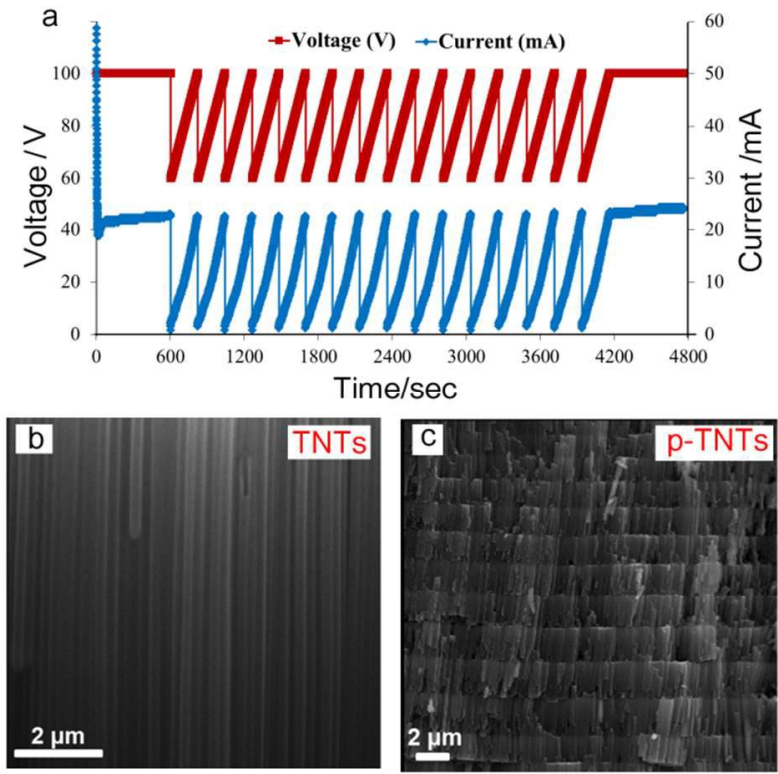

Fig. 2 Voltage/current plots and variation in structure obtained for periodic nanotubes (p-TNTs). (A) V/I plot for anodization of p-TNTs, (B-C) Cross-sectional SEM image for straight pore normal TNTs (B) and $55 \mathrm{p}$-TNTs $(\mathrm{C})$.

For better understanding of these structural changes caused by cyclic anodization process both applied voltage and recorded current signals and presented in Fig. 2a. These graphs show that signal of applied potential cycle with linear increase from $60 \mathrm{~V}$ to ${ }_{60} 100 \mathrm{~V}$ and the sudden voltage drop from $100 \mathrm{~V}$ to $60 \mathrm{~V}$ was followed with the current signal with the same shape and corresponding values from $22 \mathrm{~mA}$ to $1.5 \mathrm{~mA}$. During the increasing ramp phase ( 60 to $100 \mathrm{~V}$ ) voltage is increased at the rate of $0.22 \mathrm{~V} / \mathrm{s}$, and as a result current is also increased at the 65 rate of $0.11 \mathrm{~mA} / \mathrm{s}$. As expected these two stages have different impact on formation and dissolution of oxide layers, which disturbs the steady-state condition and hence leads to structural periodicity along the length and diameter of the nanotubes. To simplify, the sudden voltage drop reduces significantly the 70 current density and hence leads to lowered growth rates and smaller diameter, which are confirmed by top pore diameters by control experiment using this voltage. Fig. 1e can directly corelate the voltage/current oscillations with the outer diameter of nanotubes changing from $170 \pm 5 \mathrm{~nm}$ to $130 \pm 5 \mathrm{~nm}$ at every 75 single period. On other hand, the linear increase of voltage (ramp stage) results in increasing growth and oxide dissolution rate producing large diameter making asymmetrically shaped structure of this segment. After this step when the voltage drops from $100 \mathrm{~V}$ to $60 \mathrm{~V}$ the current drops from $22 \mathrm{~mA}$ to $1.5 \mathrm{~mA}$ and 80 this sudden drop (the collapse phase) disrupts the ongoing steady state and results in lowered growth rate with furthermore alteration of the pore geometry. Our results on nanostructuring of TNTs using different annodization conditions are in agreement with previous work on TNTs for solar applications where 85 nanostructuring was performed using periodic changes of current 
during anodization process [24-26].

We hypothesize that the combination of these two steps will result in conical structural pockets at each segment along the length of the nanotubes, which are seen in SEM images (Fig. 1e). ${ }_{5}$ Since characterisation of the structural modulation of internal pTNTs structure was very challenging; drug releasing characteristics were used as another argument to confirm their proposed modulated internal topography. We expect that diffusion of drug molecules on these structures will be 10 significantly altered compared with flat TNTs because of the restricted diffusion across these nanotube structures with reduced diameters in a periodic fashion which acts as barriers.
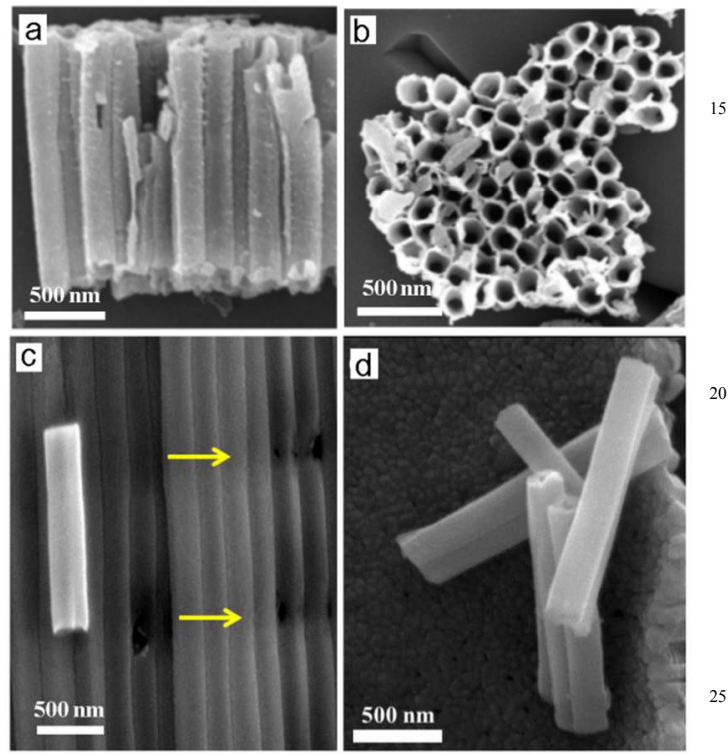

Fig. 3 (a-b) Typical structures of liberated TNTs bundles composed of hundreds joined titania nanocapsules obtained by sonication of TNTs prepared by periodic anodization. (c-d) Liberated single TNT 30 nanocapsules obtained by improved anodization process using rectangular periodic voltage $(100 \mathrm{~V}-20 \mathrm{~V}-100 \mathrm{~V})$. In both cases TNTs were openened and their potential application as micro and nano drug-carriers is proposed.

\section{Liberated nanocapsules from p-TNTs}

35 Another way to confirm the concept of periodic modulation of TNTs structures was to perform their fracturing in solution by ultrasound force. It was proposed that structural defects or weak points on the created nanotube layers by periodic anodization can be utilized to break the nanotubes into smaller fragments or single 40 nanotubes. This was the second objective of this work to confirm the preparation of liberated TNTs nanocapsules as another outcomes of this approach. However, our initial results were not very successful as we obtained liberated mixed structures including TNTs bundle, single nanotubes and their fractures.

45 Typical structures of TNTs bundles having about hundreds stacked nanotubes are presented in Figure $\mathbf{3}$ a-b. To obtain liberated single nanotubes we found that several critical parameters needs to be optimizated including weak interconnection between layers, nanotubes and mechanical force 50 during sonication process. To address the first problem we performed several trial experiments to find optimized anodization conditions able to create distinct nanotube defects on modulated segments, which could be easily broken and liberated into individual nanotubes by middle sonication. To achieve this, it 55 was found that the periodic rectangular anodization signal with high voltage differences eg. constant voltage $100 \mathrm{~V}$ for $5 \mathrm{~min}$ followed by sudden drop to $20 \mathrm{~V}$ for $1 \mathrm{~min}$ is the most effective. The TNT structure and liberated single nanotubes prepared by this method are presented in Fig. 3 c-d. When voltage is dropped 60 from $100 \mathrm{~V}$ to $20 \mathrm{~V}$, current drops from almost $20 \mathrm{~mA}$ to below $0.30 \mathrm{~mA}$. This creates vast difference in the growth rate and as a result along those periods is seen regions with partially broken or fractured nanotubes (Fig. 3c). These weak or broken spots formed at $20 \mathrm{~V}$ are utilised as breaking points for obtaining ${ }_{65}$ liberated TNT capsules with length of 1.4 to $1.7 \mu \mathrm{m}$ as presented in Figure 3d. However, some of the nanotubes are still liberated as bundle with several joined nanotubes in addition to separated individual nanotubes. The yield of individual separated nanotubes can be significantly improved by increasing the distance between

70 TNTs by varying anodization conditions for instance by increasing water content of the electrolyte. The concept of direct electrochemical synthesis of nanocapsules has been recently demonstrated by our group using pulse-anodization on $\mathrm{Al}$ in sulphuric acid and preparing alumina nanocapsule as nano75 carriers for localized delivery of anti-cancer drugs [23]. This brief investigation to obtain liberated TNTs requires further optimization by varying the anodization parameters during the periodic fabrication method to yield better control over the nanotube dimensions and improve their separation. The 80 application of these unique structures as targeted and localized drug delivery vehicles is in progress and will be reported in the following paper.

\section{Drug loading characteristics}

While the pore size and length of nanotubes was maintained the ${ }_{85}$ same for both the TNTs samples; the periodicity in p-TNTs represent small restrictions through the length which influences the drug loading amounts. From proposed model of modulated TNT structures, we can conclude (taking into consideration only the drug that was loaded inside the nanotube structures) that the 90 effective nanotube volume of p-TNTs is lower compared with flat TNTs which suggests their lower drug loading capacity. However, our TGA results confirmed contradicting results, showing that p-TNTs could load about $10 \%$ more of model drug (indomethacin) by weight compared with flat TNTs. It was 95 observed that the $\mathrm{p}$-TNTs could load more drug amount $[1.40 \mathrm{mg}$ or $17.81 \mu \mathrm{g} / \mathrm{mm}^{2}$ ] as compared to TNTs [1.28 $\mathrm{mg}$ or 16.28 $\left.\mu \mathrm{g} / \mathrm{mm}^{2}\right]$. This disagreement is explained by higher impact of the shape of internal structures of TNTs than total nanotube volume. The barriers or pockets through nanotube length served as 100 restrictions, which prevented washing of drugs from the nanotubes. The washing step during the drug loading process was necessary as it eliminates taking into consideration the high drug amount adhered to the nanotube surface (not loaded inside pores), and also can lower the initial high burst release of drug in the first 105 few hours of release study. This might not be a huge enhancement for a $1 \mathrm{~cm}$ diameter circle with nanotubes; however when scaling up to match the dimensions to actual implants, it can considerably improve the drug reservoir abilities. 

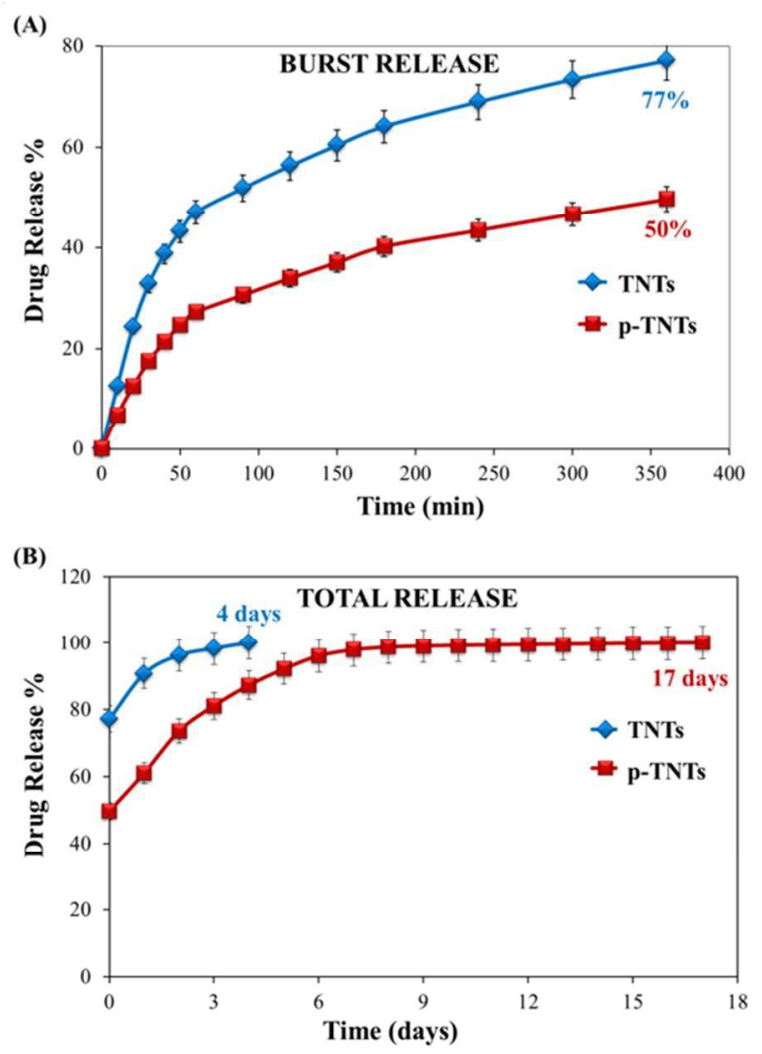

Fig. 4 In-vitro drug release graphs comparing release of model drug from conventional flat TNTs and periodic p-TNTs. (A) Initial burst release corresponding to 1 st 6 hours of drug diffusion, and (B) total drug release 5 until entire drug amount was released.

\section{Drug release characteristics}

Comparative drug release graphs of loaded drug (indomethacin) from both TNT and p-TNTs showing initial and total release are represented in Fig. 4 and Table 1. The release of drugs from 10 nanotubes represents a non-degradable drug-carrier system which is primarily diffusion-controlled (according to Fick's first law). Such systems involve mass transfer of drug molecules from region of high concentration (nanotubes) to regions of low concentration (surrounding PBS buffer). The release behaviour 15 was biphasic with initial burst release for the first 6 hours, followed by slowed release until the entire drug amount was released into the surrounding PBS. Graphs presented in Fig. 4a, show the higher burst release for TNTs about $(77 \%$ or $\sim 1 \mathrm{mg}$ ) compared with p-TNTs (only $50 \%$ or $\sim 0.7 \mathrm{mg}$ ). The burst release 20 here represents the drug amount that is present close to the open pores of the nanotubes and hence due to high concentration gradient in the initial phase, faster diffusion is expected. For a drug reservoir system such TNTs', a very high initial burst amount would mean high local drug concentration in short time, 25 which can be toxic for local tissues and also corresponds to initial wastage of loaded drug amounts. The reduced burst release for $\mathrm{p}$ TNTs is explained by their internally altered structures, which act as restricting barriers to interfere with the diffusion of drug molecules during the initial very high concentration-gradient 30 phase. Another possible explanation here is the barriers provide spaces inside the otherwise smooth straight inner pore cylinder where the drug molecules can agglomerate and adhere.

The total release patterns for both the nanotubes structures are depicted in Fig. 4b. Beyond the burst release, the diffusion of 35 drug molecules represent a slow and steady fashion; which corresponds to the zero-order release kinetics. It is again observed that the periodicity in the structure of p-TNTs plays a significant role in delaying the release of the drug from inside of the nanotube structure. For TNTs the entire drug amount was 40 released in 4 days, however for p-TNTs it was delayed till 17 days. Such zero-ordered delayed release kinetics is the most desirable therapy mechanism, where the release occurs at uniform and constant rate independent of the time and concentration [15]. Hence, the periodicity of the pocketed p-TNTs could delay the 45 therapeutic release to more than 4 times the duration for flat TNTs. This behaviour confirmed that more drug amount is left after the burst release phase; mainly due to presence of tiny pockets along the TNT length which restricted the diffusion of agglomerated drug molecules. It is significant to have prolonged

50

Table 1 Comparison of drug loading and release characteristics for model anti-inflammatory drug between TNTs and p-TNTs. p-TNTs confirmed substantial loading amounts, which were released for delayed periods while at the same time reducing initial burst release. Data are presented as 55 average of 3 measurements.

\begin{tabular}{cccc} 
& $\begin{array}{c}\text { Flat } \\
\text { TNTs }\end{array}$ & $\begin{array}{c}\text { Periodic } \\
\text { TNTs }\end{array}$ & $\begin{array}{c}\text { Improvement } \\
\text { for } \\
\text { p-TNTs }\end{array}$ \\
\cline { 2 - 4 } $\begin{array}{c}\text { Drug Loaded Amount } \\
\text { (mg) }\end{array}$ & 1.28 & 1.4 & $9 \%$ \\
\hline $\begin{array}{c}\text { Drug Loading } \\
\left.\text { Capacity ( } \mu \mathrm{g} / \mathbf{m m}^{2}\right)\end{array}$ & 16.28 & 17.81 & $9.40 \%$ \\
\hline $\begin{array}{c}\text { Initial Burst Release } \\
\text { (wt \%) }\end{array}$ & 77 & 50 & $35 \%$ \\
\hline $\begin{array}{c}\text { Total Release Time } \\
\text { (days) }\end{array}$ & 4 & 17 & $325 \%$ \\
\hline
\end{tabular}

local therapeutic effects eliminating any need to supply drugs via systemic route, and has applications more suited for bone 60 implants where a lowered drug concentration or early consumption of drugs could retrigger conditions like: bacterial adhesion/invasion.

These results clearly demonstrate how TNTs can be structurally engineered in order to enhance both their drug ${ }_{65}$ loading and release characteristics, which are more suited for bone implant applications. Previous studies using structural modifications of TNTs by controlling length and nanotube diameters where limited to improve both the characteristics at the same time. This motivated our group to explore other concept to 70 reduce the initial high burst release amounts; by employing biopolymer coating and drug encapsulation inside polymeric micelles [16,18]. Current approach is comparatively simpler considering clinical application of TNTs as drug releasing implants, which minimises additional steps and make their 75 practical applications easier. If combined with polymer coatings and micelle encapsulated drugs, $\mathrm{p}$-TNTs can provide advanced performances including further reduced initial burst release, and 
extended drug release to several months [16-18]. In this work model drug indomethacin is used but TNTs can be loaded with a variety of therapeutics like proteins, antibiotics, cancer drugs and growth factors for specific bone therapies including treatment for 5 inflammation, infection, poor bonding and primary or secondary bone cancers.

\section{Conclusions}

The improvement of drug loading and release characteristics of structurally engineered TNTs used as drug-releasing implant was 10 demonstrated by periodic modulation their nanotube structures. This process is performed by using oscillatory voltage signal (100 $\mathrm{V}$ and $60 \mathrm{~V}$ ) during anodization which provides conditions for formation of periodic segments with different nanotube diameters. It was found that more drugs can be loaded into p${ }_{15}$ TNTs than in flat TNTs even when the effective available nanotube volume of p-TNTs is less. Also, when compared with flat TNTs for in-vitro drug release, p-TNTs demonstrated better performance by showing reduced initial burst release amounts and significantly improved total release up to 17 days. These 20 improved characteristics in terms of loading and release makes pTNTs a better candidate suitable for bone implant applications compared with conventional TNTs with flat structures. In combination with other modification strategies, these new type of TNTs can be applied to address bone implant challenges like 25 bacterial infection, inflammation, poor bone-implant bonding and potentially applied for localized cancer therapy. Another outcome of presented approach is preparation of liberated titania nanocapsules, which can be explored as drug delivery nanocarriers for various targeted and localized drug delivery 30 applications.

\section{Acknowledgments}

The authors acknowledge the financial support of The Australian Research Council (FT 110100711 and DP 120101680) and The University of Adelaide for this work.

35

\section{References}

1. G. A. Rodan and T. J. Martin, Science, 2000, 289, 1508-1514.

2. K. Gulati, M. Aw, D. Findlay and D. Losic, Therapeutic delivery, 2012, 3, 857-873

40 3. P. A. Tran, L. Sarin, R. H. Hurt and T. J. Webster, Journal of Materials Chemistry, 2009, 19, 2653-2659.

4. L. Peng, A. D. Mendelsohn, T. J. LaTempa, S. Yoriya, C. A. Grimes and T. A. Desai, Nano Letters, 2009, 9, 1932-1936.

5. L. Yang and T. J. Webster, Expert Opinion on Drug Delivery, 2009, $45 \quad \mathbf{6}, 851-864$.

6. A. Kodama, S. Bauer, A. Komatsu, H. Asoh, S. Ono and P. Schmuki, Acta Biomaterialia, 2009, 5, 2322-2330.

7. T. J. Webster and J. U. Ejiofor, Biomaterials, 2004, 25, 4731-4739.

8. K. C. Popat, L. Leoni, C. A. Grimes and T. A. Desai, Biomaterials, 2007, 28, 3188-3197.

9. D. Losic and S. Simovic, Expert Opinion on Drug Delivery, 2009, 6, 1363-1381.

10. D. Losic, M. Aw, A. Santos, K. Gulati and M. Bariana, Expert Opinion on Drug Delivery, 2014 (in press)

55 11. A. Santos, M. S. Aw, M. Bariana, T. Kumeria, Y. Wang and D. Losic, Journal of Materials Chemistry B, 2014, 2, 6157-6182.

12. C. von Wilmowsky, S. Bauer, R. Lutz, M. Meisel, F. W. Neukam, T. Toyoshima, P. Schmuki, E. Nkenke and K. A. Schlegel, Journal of
Biomedical Materials Research Part B-Applied Biomaterials, 2009, 89B, 165-171.

13. K. S. Brammer, S. Oh, C. J. Cobb, L. M. Bjursten, H. van der Heyde and S. Jin, Acta Biomaterialia, 2009, 5, 3215-3223.

14. K. C. Popat, M. Eltgroth, T. J. La Tempa, C. A. Grimes and T. A. Desai, Small, 2007, 3, 1878-1881.

65 15. M. S. Aw, M. Kurian and D. Losic, Biomaterials Science, 2014, 2 , 10-34.

16. K. Gulati, S. Ramakrishnan, M. Aw, G. Atkins, D. Findlay and D. Losic, Acta biomaterialia, 2012, 8, 449-456.

17. S. Simovic, D. Losic and K. Vasilev, Chemical Communications, 2010, 46, 1317-1319.

18. M. S. Aw, K. Gulati and D. Losic, Journal of Biomaterials and Nanobiotechnology, 2011, 2, 477-484.

19. G. Gao, D. Lange, K. Hilpert, J. Kindrachuk, Y. Zou, J. T. J. Cheng, M. Kazemzadeh-Narbat, K. Yu, R. Wang, S. K. Straus, D. E.

75 Brooks, B. H. Chew, R. E. W. Hancock and J. N. Kizhakkedathu, Biomaterials, 2011, 32, 3899-3909.

20. M. Kazemzadeh-Narbat, B. Lai, C. Ding, J. Kizhakkedathu, R. Hancock and R. Wang, Biomaterials, 2013, 34, 5969-5977.

21. D. Losic, M. Lillo and D. Losic, Jr., Small, 2009, 5, 1392-1397.

80 22. G.K. Mor, O. K. Varghese, M. Paulose, N.Mukherjee and C. A. Grimes, Journal of Materials Research, 2003, 18, 2588-2593.

23. Y. Wang, A. Santos, G. Kaur, A. Evdokiou and D. Losic, Biomaterials, 2014, 35, 5517-5526.

24. J. Lin, K. Liu and X. Chen, Small, 2011, 7, 1784-1789.

85 25. Y. Liu, D. Wang, L. Cao and S. Chen, Electrochemistry Communications, 2012, 23, 68-71.

26. P. Roy, S. Berger and P. Schmuki, Angewandte Chemie-International Edition, 2011, 50, 2904-2939.

\section{${ }_{90}$ Graphical Abstract:}

Structurally engineering of titania nanotubes (TNTs) with periodically shaped structures was successfully demonstrated as a strategy to improve drug loading and releasing performances of drug-implants

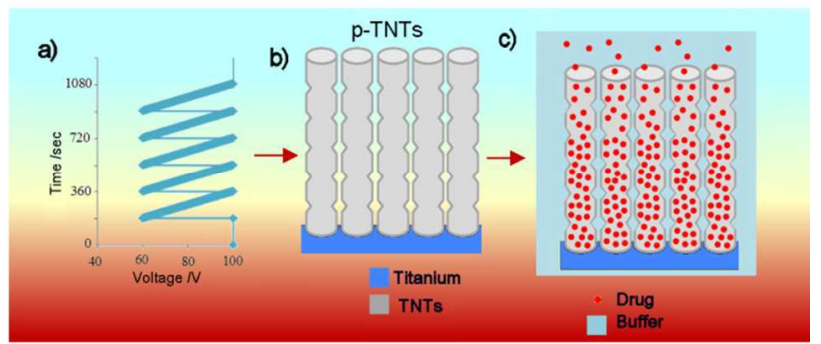




\section{Journal of \\ Materials Chemistry B}

Accepted Manuscript

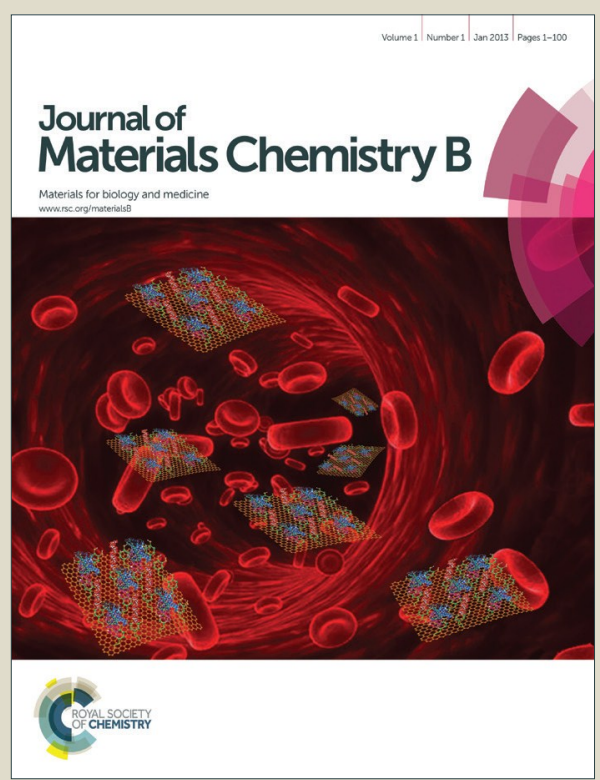

This is an Accepted Manuscript, which has been through the Royal Society of Chemistry peer review process and has been accepted for publication.

Accepted Manuscripts are published online shortly after acceptance, before technical editing, formatting and proof reading. Using this free service, authors can make their results available to the community, in citable form, before we publish the edited article. We will replace this Accepted Manuscript with the edited and formatted Advance Article as soon as it is available.

You can find more information about Accepted Manuscripts in the Information for Authors.

Please note that technical editing may introduce minor changes to the text and/or graphics, which may alter content. The journal's standard Terms \& Conditions and the Ethical guidelines still apply. In no event shall the Royal Society of Chemistry be held responsible for any errors or omissions in this Accepted Manuscript or any consequences arising from the use of any information it contains. 